\title{
Simultaneous formation of surface ripples and metallic nanodots induced by phase decomposition and focused ion beam patterning
}

\author{
Jie Lian ${ }^{\text {a) }}$ \\ Department of Geological Sciences, University of Michigan, Ann Arbor, Michigan 48109 \\ Wei Zhou \\ School of Mechanical and Aerospace Engineering, Nanyang Technological University, Singapore 639798 \\ Q. M. Wei and L. M. Wang \\ Department of Nuclear Engineering and Radiological Sciences, University of Michigan, Ann Arbor, \\ Michigan 48109 \\ L. A. Boatner \\ Oak Ridge National Laboratory, Oak Ridge, Tennessee 37831 \\ Rodney C. Ewing \\ Departments of Geological Sciences, Materials Science and Engineering, and Nuclear Engineering \\ and Radiological Sciences, University of Michigan, Ann Arbor, Michigan 48109
}

(Received 8 November 2006; accepted 17 January 2006; published online 1 March 2006)

\begin{abstract}
We report the simultaneous formation of self-assembled surface ripples in $\mathrm{Cd}_{2} \mathrm{Nb}_{2} \mathrm{O}_{7}$ pyrochlore caused by focused ion beam (FIB) patterning and uniformly distributed metallic nanodots induced by phase decomposition under ion bombardment. The characteristic wavelength of the surface ripples is controllable from the $\mathrm{nm}$ to the sub- $\mu \mathrm{m}$ scale. High-density $\mathrm{Cd}$ metallic nanoparticles, $\sim 5 \mathrm{~nm}$, formed and the distribution of nanoparticles is consistent with the morphological characteristics of the ripple pattern. This approach provides a means of fabricating surface nanostructure with various patterns and a controllable particle size and distribution by combining ion beam-induced phase decomposition with high-precision FIB patterning. (C) 2006 American Institute of Physics. [DOI: 10.1063/1.2181203]
\end{abstract}

Ion bombardment of solid surfaces can result in the formation of periodic ripples or wavelike patterns with a spatial periodicity varying from the $\mathrm{nm}$ to the $\mu \mathrm{m}$ range. The spontaneous formation of self-assembled rippled patterns can be understood in terms of the instability caused by surfacecurvature-dependent sputtering. ${ }^{1,2}$ The competition between the surface roughing process due to ion sputtering and a diffusion process generates ripples with a characteristic wavelength depending on the surface diffusivity and the ion erosion rate. Ripple structures have been observed in many materials, such as crystalline or amorphous $\mathrm{SiO}_{2},{ }^{3}$ metals (e.g., $\mathrm{Ag},{ }^{4} \mathrm{Cu}^{5}{ }^{5}$ graphite, ${ }^{6}$ and diamond, ${ }^{7}$ and semiconductors (e.g., $\mathrm{Ge}^{8} \mathrm{Si}^{9}$ ). Recently, ion-induced ripple structures have been of particular interest for the fabrication of nanoscale-textured materials via self-organization processes or as templates for the growth of nanowires, nanorods, and nanodots. ${ }^{10}$ A regular hexagonal dot pattern $(35 \mathrm{~nm}$ in diameter) has been created on GaSb surface by ion beam sputtering. ${ }^{11}$ Similarly, ordered silicon nanostructures have been produced by ion sputtering at normal incidence. ${ }^{12}$ These results demonstrated the potential application of using ion sputtering method for device fabrication.

In this letter, we report the experimental results for the simultaneous formation of a ripple structure with a characteristic wavelength, varying from the $\mathrm{nm}$ to the sub- $\mu \mathrm{m}$ range, induced by ion sputtering and the formation of uniformly distributed metallic nanodots, $\sim 5 \mathrm{~nm}$ in size, caused by ion irradiation-induced phase decomposition. The surface

\footnotetext{
a) Author to whom correspondence should be addressed; electronic mail: jlian@umich.edu
}

ripple patterns were created by focused ion beam (FIB) patterning, and the distribution of high-density metallic nanoparticles follows the morphological characteristics of the surface ripple patterns. This method provides an approach to fabricate surface nanostructure with various patterns and a controllable size and distribution in similar materials (e.g., zircon, GaN, or GaAs) by combining irradiation-induced phase decomposition and high-precision FIB patterning.

The FIB bombardment experiments were performed on $\mathrm{Cd}_{2} \mathrm{Nb}_{2} \mathrm{O}_{7}$ pyrochlore single crystals. Pyrochlore compounds, $\mathrm{A}_{2} \mathrm{~B}_{2} \mathrm{O}_{7}$, display an unusual variety of physical, chemical, and electronic properties due to the remarkable range of compositions and extensive cation substitutions at both the A and B sites, ${ }^{13}$ and they are important in numerous technological applications that encompass catalysts, piezoelectric materials, ferro- and ferrimagnetism, luminescent compounds, giant magnetoresistant materials, and as a solid electrolyte in solid oxide fuel cells. ${ }^{14}$ Importantly, the properties of pyrochlore, e.g., the ionic conductivity, can be manipulated on a nanometer scale by the use of ion-beam irradiation or implantation techniques, and numerous nanostructures_including nanodots and nanodomains, ${ }^{15}$ one-dimensional nanowires, ${ }^{16}$ and two-dimensional nanolayers ${ }^{17}$ - have been created by ion beam techniques in pyrochlore materials.

The single-crystal wafers of $\mathrm{Cd}_{2} \mathrm{Nb}_{2} \mathrm{O}_{7}$ with a $\langle 111\rangle$ orientation used in this study were grown by means of a hightemperature solvent (flux) technique. ${ }^{18}$ The ion bombardment experiments were performed using a field emission scanning electron microscopy (SEM)/FIB dual-beam system (FEI Nova 200 NanoLab). A $30 \mathrm{keV}$ focused $\mathrm{Ga}^{+}$beam was used 

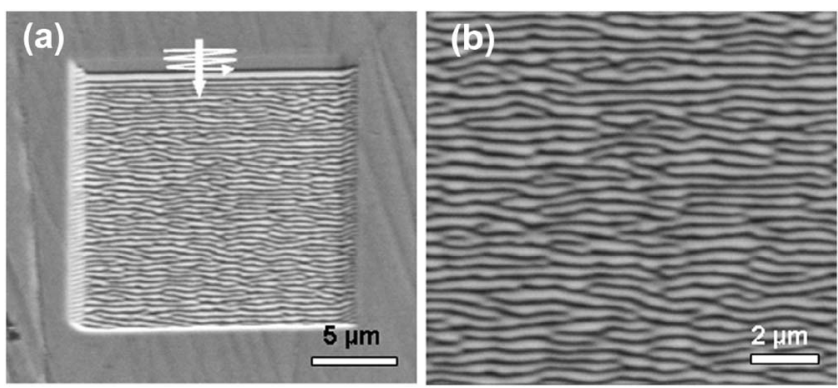

FIG. 1. (a) A SEM image showing the ripples created by $30 \mathrm{keV} \mathrm{Ga}^{+}$focused ion beam bombardment on a $\mathrm{Cd}_{2} \mathrm{Nb}_{2} \mathrm{O}_{7}$ single crystal surface (ion current, $0.3 \mathrm{nA}$; incident angle, $50^{\circ}$; patterned area, $15 \times 10 \mu \mathrm{m}^{2}$; and ion fluence of $5.38 \times 10^{17}$ ions $/ \mathrm{cm}^{2}$ ). The orientation of the ripples is perpendicular to the ion projection direction. The curved arrow indicates the FIB scanning direction, and the straight arrow indicates the ion projection direction. The characteristic wavelength of ripples is $\sim 335 \mathrm{~nm}$, as measured from the close up image (b).

for ion sputtering with varying beam currents from $10 \mathrm{pA}$ to $5 \mathrm{nA}$ at different incident angles. Compared with broad ion beam irradiation, the FIB allows in situ observation of the formation of ripple structures in real time using a scanning electron beam or ion beam imaging. The FIB raster over a predesigned rectangular area at an incident angle of $50^{\circ}$ at a dwell time of $1 \mu \mathrm{s}$, and the beam was scanned over the area using as many passes as required to achieve the desired total ion fluence. Figures 1(a) and 1(b) show the SEM images of a typical ripple structure patterned by $\mathrm{FIB}$ on the $\mathrm{Cd}_{2} \mathrm{Nb}_{2} \mathrm{O}_{7}$ surface. The average wavelength as measured from the SEM image, is $\sim 335 \mathrm{~nm}$, and the uncertainty in the measurements is estimated to be $\sim 5 \%$. The characteristic ripple wavelength can be controlled from the $\mathrm{nm}$ scale $(\sim 130 \mathrm{~nm})$ to the submicron scale $(\sim 550 \mathrm{~nm})$, varying with different irradiation fluences.

Surface ripples were patterned at a thin transmission electron microscopy (TEM) foil preprepared by ion milling, which allow us to characterize the microstructure at the atomic scale. Figure 2(a) shows the morphology of the surface ripple with the characteristic wavelength $\sim 130 \mathrm{~nm}$. The microstructure of the patterned ripple structure was investigated in details using a JEOL $2010 \mathrm{~F}$ analytic transmission electron microscope with a field emission source operated at $200 \mathrm{kV}$. The ripple structure was clearly evident by the highangle annular dark-field scanning transmission electron microscopy imaging (Z-contrast image) [Fig. 2(b)]. The rippled
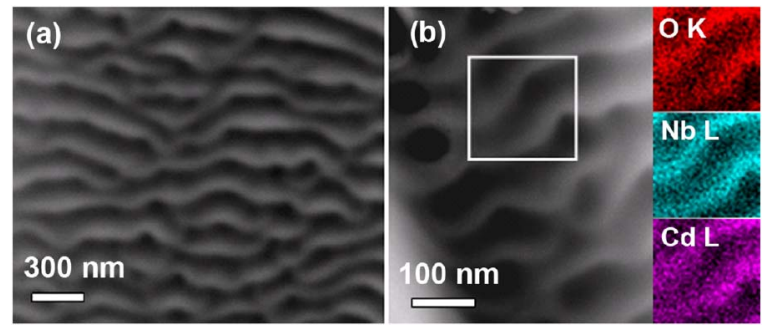

FIG. 2. (a) A SEM image showing the morphology of ripple structure $\left(4 \times 3 \mu \mathrm{m}^{2}\right)$ patterned by $30 \mathrm{keV} \mathrm{Ga}^{+}$ions at a thin TEM foil (beam current, $10 \mathrm{pA}$; incident angle, $50^{\circ}$ ). The total fluence experienced by this patterned area is $4.3 \times 10^{18}$ ions $/ \mathrm{cm}^{2}$. (b) The Z-contrast TEM image showing the contrast variation due to the height modulation in which the crest of ripple has brighter contrast. The EDS elemental mapping [inset in (b)] acquired from the area outlined by the white box showing a chemical composition variation across the ripples.
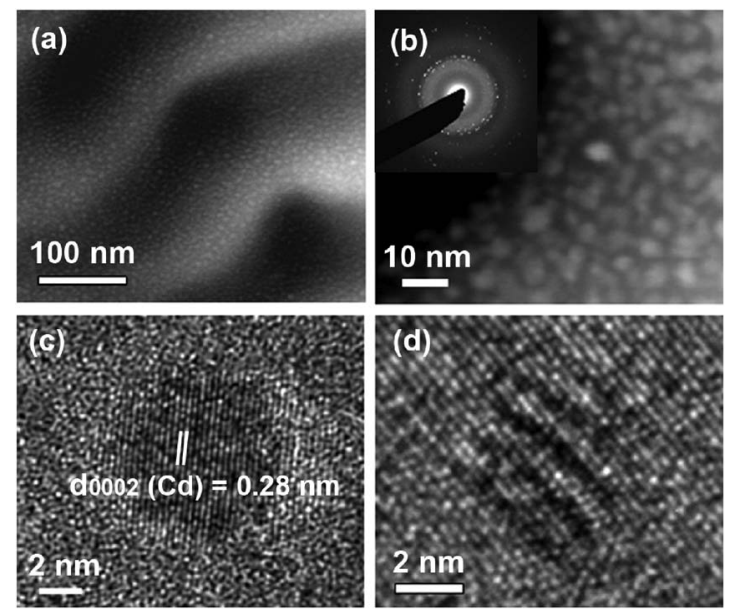

FIG. 3. Z-contrast images [(a) and (b)] showing the formation of highdensity nanoparticles. The nanoparticles exhibit a high uniformity in particle size $(\sim 5 \mathrm{~nm})$ and the distribution of nanoparticles follows the characteristic morphology of the surface ripple patterns. The inset in (b) is the selectedarea electron diffraction pattern acquired from the edge of the thin area, and the nanoparticles can be indexed as pure Cd-metal. High-resolution TEM images show the nanoparticles embedded in the surface amorphous region (c) and on top of the crystalline substrate (d).

area has a significant variation in contrast, resulting from the height modulation of the ripple structure. The crests of the ripples have a brighter contrast due to their greater sample thickness. Energy dispersive spectroscopy (EDS) elemental maps [inset in Fig. 2(b)] acquired in the scanning transmission electron microscopy mode from the region outlined by the white square exhibit a wavelike variation that follows the periodicity of the ripples. High-density nanoparticles showing bright contrast were observed in the $Z$-contrast images [Figs. 3(a) and 3(b)]. Interestingly, the nanoparticles are uniformly distributed, following the morphological characteristics of the ripple pattern, as the height modulates across the crest of the ripple. The size of the nanoparticles is estimated to be $\sim 5 \mathrm{~nm}$ as shown in the $Z$-contrast and high-resolution TEM images [Fig. 3(c)]. EDS measurements indicated that these nanoparticles are $\mathrm{Cd}$-rich but depleted in $\mathrm{Nb}$ and $\mathrm{O}$. The nanoparticles were identified as pure $\mathrm{Cd}$ metal by indexing the ring pattern [inset in Fig. 3(b)] and the lattice image [Fig. 3(c)].

Using the SRIM-2003 code and assuming a threshold displacement energy of $50 \mathrm{eV}$, the ion projected range of $30 \mathrm{keV} \mathrm{Ga}{ }^{+}$ions in $\mathrm{Cd}_{2} \mathrm{Nb}_{2} \mathrm{O}_{7}$ at an incident angle of $50^{\circ}$ was predicted to be $\sim 9.6 \mathrm{~nm}$. $\mathrm{Cd}_{2} \mathrm{Nb}_{2} \mathrm{O}_{7}$ can be amorphized at room temperature at a relatively low fluence (e.g., $1.11 \times 10^{15}$ ions $/ \mathrm{cm}^{2}$ using $280 \mathrm{keV} \mathrm{Ne}{ }^{+}$ion irradiation). ${ }^{18}$ Therefore, an amorphous surface region with the depth at which the damage level is above the critical dose was created by the $30 \mathrm{keV} \mathrm{Ga}^{+}$ion bombardment, and this region is responsible for the diffuse scattering halo observed in the diffraction pattern. Some nanoparticles were embedded in the amorphous region [Fig. 3(c)]. A high-resolution TEM image [Fig. 3(d)] acquired from thicker area shows a nanoparticle located at top of the crystalline substrate, as evidenced by the Moire fringe. This result indicates that the nanoparticles were only confined in the amorphous surface region, while the unirradiated substrate, beyond the ion range of $\mathrm{Ga}^{+}$in $\mathrm{Cd}_{2} \mathrm{Nb}_{2} \mathrm{O}_{7}$, remained crystalline.

The Cd nanoparticles are formed by a phase decomposition process induced by energetic ion bombardment. The 
irradiation-induced formation of $\mathrm{Cd}$ nanoparticles with the size of $\sim 20 \mathrm{~nm}$ has been previously reported to occur ran-

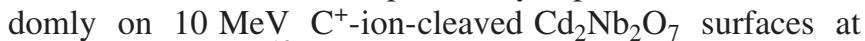
room temperature, ${ }^{19}$ and the average particle size increases up to $150 \mathrm{~nm}$ with both increasing ion fluence and deposition energy density. An unusually high intensity of luminescence in the visible range was observed as the nanoparticle-rich surface was exposed to the ion beam irradiation, as compared with the original $\mathrm{Cd}_{2} \mathrm{Nb}_{2} \mathrm{O}_{7}$ single crystal. ${ }^{19}$ The nanoparticle formation also occurred for $1 \mathrm{MeV}$ $\mathrm{Kr}^{2+}$-irradiated $\mathrm{Cd}_{2} \mathrm{Nb}_{2} \mathrm{O}_{7} \cdot{ }^{20}$ The soft metal $\mathrm{Cd}$ segregates in the phase decomposed region under ion bombardment, and may then nucleate and grow in the amorphous region. The energetic ion beam irradiation may enhance the migration of $\mathrm{Cd}$, and smaller nanoparticles may coalesce resulting in the formation of larger particles at higher irradiation fluences or at higher temperatures. A large number of nanopores were created nearby as the $\mathrm{Cd}$ nanoparticles segregated out of the matrix. ${ }^{19,20}$ A $3 \mathrm{MeV} \mathrm{He}^{+}$ion-irradiation created $\mathrm{Cd}$ nanowires with a length up to several tens of microns and a diameter ranging from less than $100 \mathrm{~nm}$ to nearly $1 \mu \mathrm{m} .{ }^{16}$

A completely different morphology was observed on the FIB bombarded surface even though the patterned area (Fig. 3) experienced a total fluence as high as $4.3 \times 10^{18}$ ions $/ \mathrm{cm}^{2}$. Uniformly distributed $\mathrm{Cd}$ nanoparticles, $\sim 5 \mathrm{~nm}$ in size, were observed, suggesting that the size of nanoparticles does not increase significantly at higher irradiation fluence. As the surface material was sputtered away, a fresh surface was exposed during the FIB sputtering process. Focused $\mathrm{Ga}^{+}$ion bombardment caused the phase decomposition of $\mathrm{Cd}_{2} \mathrm{Nb}_{2} \mathrm{O}_{7}$ and produced high-density nucleation centers on the irradiated surface region. Due to the shallow projected range $\left(\sim 9.6 \mathrm{~nm}\right.$ at an incident angle of $\left.50^{\circ}\right)$ of $30 \mathrm{keV} \mathrm{Ga}+$ ions in $\mathrm{Cd}_{2} \mathrm{Nb}_{2} \mathrm{O}_{7}$, decomposition only occurred in the surface region. Limited amounts of free metallic $\mathrm{Cd}$ were available for migration, and the coalescence of small nanoparticles could not occur. In contrast, in highly energetic ion experiments, the projected ion range varied from hundreds of nm (e.g., $1 \mathrm{MeV} \mathrm{Kr}^{2+}$ ) and up to several $\mu \mathrm{m}$ (e.g., $10 \mathrm{MeV} \mathrm{C}^{+}$and $3 \mathrm{MeV} \mathrm{He}^{+}$), and extensive amount of materials were decomposed depending on ion fluence, leading to the coalescence of nanoparticles and a significant increase in particle size upon higher ion fluence. ${ }^{19}$ Therefore, the uniformity in the nanoparticle size in this study was achieved by controlling the projected ion range of the incident FIBs and thus limit amount of materials decomposed for nanoparticle nucleation and growth.

In summary, we report the simultaneous formation of self-assembled ripples caused by ion sputtering and uniformly distributed metallic nanoparticles, $\sim 5 \mathrm{~nm}$, caused by ion irradiation-induced decomposition. The characteristic wavelength of the ripples can be controlled from the $\mathrm{nm}$ to the sub- $\mu \mathrm{m}$ scale. Metallic nanoparticles exhibiting an excellent uniformity in the particle size, $\sim 5 \mathrm{~nm}$, were formed and confined in the surface region. Various patterns can actually be achieved by the high-precision FIB patterning, and we demonstrate that the distribution of the nanoparticles follows the characteristic morphologies of the surface pattern. The method described above may be applicable to similar materials (e.g., zircon, GaN, or GaAs) for controllable and reproducible fabrication of surface nanostructures with various patterns and controllable size and distribution by utilizing the unique capability of high-precision focus ion beam patterning.

This work was supported by the Office of Basic Energy Science of the U.S. Department of Energy, through Grant No. DE-FG02-97ER45656 and the NSF NIRT program (EAR-0309772). Oak Ridge National Laboratory is managed by UT-Battelle, LLC, for the USDOE under Contract No. DE-AC05-00OR22725.

${ }^{1}$ R. M. Bradley and J. M. E. Harper, J. Vac. Sci. Technol. A 6, 2390 (1988).

${ }^{2}$ R. Cuerno and A.-L. Barabási, Phys. Rev. Lett. 74, 4746 (1995).

${ }^{3}$ T. M. Mayer, E. Chason, and A. J. Howard, J. Appl. Phys. 76, 1633 (1994).

${ }^{4}$ S. Rusponi, C. Boragno, and U. Valbusa, Phys. Rev. Lett. 78, 2795 (1997).

${ }^{5}$ S. Rusponi, G. Costantini, C. Boragno, and U. Valbusa, Phys. Rev. Lett. 81, 2735 (1998).

${ }^{6}$ S. Habenicht, W. Bolse, K. P. Lieb, K. Reimann, and U. Geyer, Phys. Rev. B 60, R2200 (1999).

${ }^{7}$ A. Datta, Y.-R. Wu, and Y. L. Wang, Phys. Rev. B 63, 125407 (2001).

${ }^{8}$ E. Chason, T. M. Mayer, B. K. Kellerman, D. T. McIlroy, and A. J. Howard, Phys. Rev. Lett. 72, 3040 (1994).

${ }^{9}$ (a) J. Erlebacher, M. J. Aziz, E. Chason, M. B. Sinclari, and J. A. Floro, Phys. Rev. Lett. 82, 2330 (1999); (b) J. Erlebacher, M. J. Aziz, E. Chason, M. B. Sinclair, and J. A. Floro, J. Vac. Sci. Technol. A 18, 115 (2000).

${ }^{10}$ U. Valbusa, C. Boragno, and F. B. de Mongeot, J. Phys.: Condens. Matter 14, 8153 (2002).

${ }^{11}$ S. Facsko, T. Dekorsy, C. Koerdt, C. Trappe, H. Kurz, A. Vogt, and H. L. Hartnagel, Science 285, 1551 (1999).

${ }^{12}$ R. Gago, L. Vazquez, R. Cuerno, M. Varela, C. Ballesteros, and J. M. Albella, Appl. Phys. Lett. 78, 3316 (2001).

${ }^{13}$ A. Subramanian, G. Aravamudan, and G. V. S. Rao, Prog. Solid State Chem. 15, 55 (1983).

${ }^{14}$ (a) P. K. Moon and H. L. Tuller, Solid State Ionics 28, 470 (1988); (b) B. J. Wuensch and K. W. Eberman, JOM 52, 19 (2000); (c) H. L. Tuller, Solid State Ionics 52, 135 (1992).

${ }^{15}$ J. Lian, X. T. Zu, K. V. G. Kutty, J. Chen, L. M. Wang, and R. C. Ewing, Phys. Rev. B 66, 054108 (2002).

${ }^{16}$ W. Jiang, W. J. Weber, C. M. Wang, J. S. Young, L. A. Boatner, J. Lian, L. M. Wang, and R. C. Ewing, Adv. Mater. (Weinheim, Ger.) 17, 1602 (2005).

${ }^{17}$ J. Lian, L. M. Wang, S. X. Wang, J. Chen, L. A. Boatner, and R. C. Ewing, Phys. Rev. Lett. 87, 145901 (2001).

${ }^{18}$ A. Meldrum, C. W. White, V. Keppens, L. A. Boatner, and R. C. Ewing, Phys. Rev. B 63, 104109 (2001).

${ }^{19}$ W. Jiang, W. J. Weber, J. S. Young, and L. A. Boatner, Appl. Phys. Lett. 80, 670 (2002).

${ }^{20}$ J. Lian, L. M. Wang, L. A. Boatner, and R. C. Ewing (unpublished). 